\title{
Variation, emprunt, remploi au cœur de l'écriture enfantine
}

Variation, borrowing, quotation about pupils writing process

\section{Pierre Sève}

\section{(2) OpenEdition \\ 1 Journals}

\section{Electronic version}

URL: http://journals.openedition.org/pratiques/3372

DOI: 10.4000/pratiques.3372

ISSN: 2425-2042

\section{Publisher}

Centre de recherche sur les médiations (CREM)

\section{Electronic reference}

Pierre Sève, « Variation, emprunt, remploi au cœur de l'écriture enfantine », Pratiques [Online], 173-174 | 2017, Online since 22 March 2017, connection on 14 November 2019. URL : http:// journals.openedition.org/pratiques/3372; DOI : 10.4000/pratiques.3372

This text was automatically generated on 14 November 2019.

(c) Tous droits réservés 


\title{
Variation, emprunt, remploi au cœur de l'écriture enfantine
}

\author{
Variation, borrowing, quotation about pupils writing process
}

\section{Pierre Sève}

Ce qui est déjà dit t'est donné :

Le monde et son mot de passe.

François Cheng, À l'orient de tout

1 Les jeunes enfants acquièrent le langage en imitant celui de leur entourage. Chez les artistes, il n'est pas rare que les débutants forment leur métier dans l'imitation des œuvres de leurs prédécesseurs qu'ils reproduisent ou pastichent. Pourtant, dans les classes, c'est souvent un regard dépréciatif qu'on porte envers la copie ou l'imitation et nombre de maitres se désolent de travaux honorables, mais « qui ne disent pas grandchose » tant ils paraissent meublés de stéréotypes ${ }^{1}$.

Plusieurs raisons contribuent à cet état de fait. D'abord, la copie de modèles est une activité centrale dans l'acquisition du geste graphique ; elle peut apparaitre comme une activité " de petits ", même quand le scripteur porte la responsabilité de la sélection du morceau à copier. Ensuite, l'engagement subjectif dans la parole tenue, qui commence à s'étoffer quand débute l'apprentissage de l'écriture, constitue une expérience que l'enfant peut vouloir retrouver dans la production de textes; et cette référence à l'échange oral peut se trouver renforcée de ce que la perception des relations grapho-phonologiques et l'entrainement à l'oralisation ont vite fait de présenter l'écriture comme le reflet, voire le double laborieux, d'un discours oral. Enfin, les modèles didactiques issus des travaux du groupe EVA (1991, 1993) qui, à notre connaissance, dominent les pratiques d'enseignement effectives dans les classes promeuvent une interaction entre lecture et écriture d'une grande complexité - à vrai dire, d'une complexité telle qu'elle assèche chez certains maitres l'ambition même d'enseigner l'écriture.

Dans ces modèles les textes proposés en amont, dûment sélectionnés autour d'un apparentement de leur enjeu pragmatique, servent à identifier cet enjeu et font la base 
d'une écriture dont le projet est de le réaliser dans les productions envisagées: la formulation de la consigne objectivera le but à atteindre. Ensuite, la lecture de ces textes permet d'en dégager les invariants, lesquels invariants trouvent leur représentation dans une sorte d'expansion de la consigne (liste des critères de réussite, grille de relecture...). Enfin, les textes lus constituent un répertoire de moyens linguistiques et rhétoriques où les jeunes scripteurs pourront à loisir piocher des modèles pour son propre texte, à la condition de servir un projet autonome. Cette logique se caractérise par deux traits qui nous semblent trouver leurs limites. D'une part, l'interaction lecture/écriture n'est pensée qu'entre les textes lus en amont et le texte produit en aval - sans grande considération pour l'interaction entre l'écriture et la lecture des textes produits; cela conduit à faire pratiquer l'écriture dans une perspective de mise en conformité avec les canons dégagés, et non pas du tout dans une perspective de tâtonnement et d'exploration plus ou moins heureuse en vue d'être lu. D'autre part, c'est négliger les différences qui peuvent se faire jour entre une pratique experte qui mobilise stratégiquement des références dont l'opérationnalité est éprouvée, et une pratique débutante qui bricole plus ou moins ingénieusement avec les références qui sont déjà là. De fait, une telle démarche suppose une adhésion au projet de servir un enjeu pragmatique similaire et non identique à celui des textes lus en amont ; le plus souvent, il n'est pas seulement envisagé d'aider à construire un tel projet alors même que la moindre expérience d'une écriture apprend combien son projet s'éclaire et s'éprouve en relation avec les mots déjà tracés et ceux qu'on cherche. Les élèves, du moins les plus fragiles ou ceux qui paraissent encore peu familiers de la culture scolaire et des marges de liberté qu'elle autorise, peuvent se sentir pris dans une injonction paradoxale : sois conforme, et sois toi-même.

4 Pour identifier quelles pourraient être des interventions utiles, il nous semble opportun d'étudier sur quels points d'appui comptent les élèves pour risquer l'aventure de l'écriture. De très nombreux travaux - si nombreux qu'on ne saurait tous les citer - se sont déjà intéressés aux productions enfantines; cependant ces études portent le plus souvent sur des écrits commandés, parfois formatés par des situations scolaires, ou alors sur des écrits considérés pour eux-mêmes, sans considération d'un quelconque contexte intellectuel ou culturel. Pour notre part, nous étudions ici des productions singulières, qui se situent à la périphérie de la prescription scolaire au sens où les élèves n'étaient pas en situation d'identifier clairement ce qui aurait été attendu.

\section{Les données recueillies}

5 Les écrits recueillis viennent d'une classe ${ }^{2}$ qui a lu un réseau complexe construit autour du motif du mur: le mur qu'on franchit, le mur qu'on construit et le mur qu'on contemple. Nous avons exposé ailleurs (Sève, 2004) l'ensemble des séquences où s'est déployé le travail. Nous rappellerons ici seulement les œuvres lues et les traces où la classe avait formalisé une modélisation du motif.

\subsection{Un réseau de lecture « à tiroir »}

6 Dans un premier temps, la classe avait lu Tillie et le mur, de L. Lionni (1989), Le Jardin de Max et Gardénia, de F. Bernard et F. Roca (1998), Volubilis et les trois jardins, de C. Bernos (1986) et Le Mur d'A. Esteban (1991). Les élèves avaient perçu comment la frontière d'un mur pouvait engendrer des fantasmes, qu'elle enserre un paradis comme une cage dorée, 
qu'elle constitue un passage initiatique au delà des apparences ou qu'elle figure la frontière infranchissable de la mort... Voici le tableau qui résumait la configuration mise en lumière.

Tableau 1.Les murs à franchir

\begin{tabular}{|l|l|l|l|l|}
\hline & en deçà & au delà imaginé & au delà rencontré & $\begin{array}{l}\text { au delà du } \\
\text { deuxième mur } \\
\text { imaginé }\end{array}$ \\
\hline $\begin{array}{l}\text { Tillie et le } \\
\text { mur }\end{array}$ & $\begin{array}{l}\text { monde } \\
\text { ordinaire }\end{array}$ & $\begin{array}{l}\text { veut voir un monde } \\
\text { extraordinaire }\end{array}$ & $\begin{array}{l}\text { monde ordinaire, mais un } \\
\text { caillou de cérémonie }\end{array}$ & - non pertinent - \\
\hline $\begin{array}{l}\text { Jardin de Max } \\
\text { et Gardénia } \\
\text { dorée }\end{array}$ & $\begin{array}{l}\text { Max a peur de ce qui } \\
\text { vient d'au delà }\end{array}$ & $\begin{array}{l}\text { Max n'a plus peur : ce qui } \\
\text { faisait peur n'était que des } \\
\text { apparences }\end{array}$ & - non pertinent - \\
\hline $\begin{array}{l}\text { Volubilis et } \\
\text { les trois } \\
\text { jardins }\end{array}$ & ennui & $?$ & $\begin{array}{l}\text { jardins luxuriants } \\
\text { - un nain } \\
\text { - un jardinier } \\
\text { - Zoé }\end{array}$ & $\begin{array}{l}\text { ce qu'on ne peut } \\
\text { atteindre : } \\
\text { des gens } \\
\text { très loin } \\
\text { on imagine }\end{array}$ \\
\hline $\begin{array}{l}\text { Le Mur } \\
\text { monde }\end{array}$ & $?$ & $\begin{array}{l}\text { Lamité } \\
\text { Le paradis? }\end{array}$ & - non pertinent - \\
\hline
\end{tabular}

7 Plus tard dans l'année, les élèves ont lu deux variations autour de l'histoire des Trois Petits Cochons, l'une très proche du dessin animé qu'en ont tiré les studios de Walt Disney, et une autre, japonaise, qui présente le cochon prévoyant satisfait d'être débarrassé de ses frères. Ils ont lu aussi Les Trois Petites Cochonnes, une parodie de F. Stehr (1997). Ils ont dégagé les valeurs idéologiques associées :

Tableau 2. Version du dessin animé

\begin{tabular}{|l|l|l|}
\hline mur & caractère du personnage & issue \\
\hline paille : naturellement pas très solide, pas cher & paresseux & négative \\
\hline bois : « aux prises avec une scie » presque aussi fragile & joueur & négative \\
\hline briques : « superposer les briques avec soin » & prudent & positive \\
\hline
\end{tabular}

Tableau 3. Version japonaise

\begin{tabular}{|l|l|l|}
\hline mur & caractère du personnage & issue \\
\hline
\end{tabular}




\begin{tabular}{|l|l|l|}
\hline $\begin{array}{l}\text { paille : «aussitôt dit, } \\
\text { aussitôt fait » }\end{array}$ & $\begin{array}{l}\text { On n'en sait rien : on a l'impression qu'ils ne choisissent } \\
\text { pas, c'est par hasard }\end{array}$ & négative \\
$\begin{array}{l}\text { bois : « aussitôt dit, aussitôt } \\
\text { fait » }\end{array}$ & négative \\
\hline $\begin{array}{l}\text { briques : «aussitôt dit, } \\
\text { presque fait » }\end{array}$ & rusé & positive \\
\hline
\end{tabular}

Tableau 4. Version parodique

\begin{tabular}{|l|l|l|}
\hline mur & caractère du personnage & issue \\
\hline $\begin{array}{l}\text { pierres : « dépense tout son sac d'or pour acheter une } \\
\text { grosse maison » }\end{array}$ & $\begin{array}{l}\text { aime le confort et le « tape à } \\
\text { l'œil » }\end{array}$ & négative \\
\hline $\begin{array}{l}\text { bois : « se fait construire pour la moitié du sac d'or une } \\
\text { belle cabane» }\end{array}$ & aime le « peinard » & négative \\
\hline paille : insouciance & goût simple + ruse & positive \\
\hline
\end{tabular}

Dans un troisième temps, la classe a lu Le Livre de la Lézarde, d'Y. Heurté (1998). Les élèves ont dégagé que le mur s'offrait comme un écran pour déployer la fantasmagorie propre au personnage qui le contemple.

Enfin, pour conclure ce parcours, la maitresse a soumis un ensemble de phrases avec cette consigne : «Voici des phrases extraites de sept histoires. À ton avis, à quel type d'histoire chacune appartient-elle? Est-ce une histoire de mur qu'on franchit? de mur qu'on construit? de mur qu'on regarde?»

10 Sans avoir fourni de réponse définitive à cette consigne - mais ce n'était pas le but poursuivi - les élèves ont synthétisé leurs remarques dans le tableau suivant :

Tableau 5. Figures de mur

\begin{tabular}{|c|c|c|}
\hline le mur qu'on franchit & le mur qu'on construit & le mur qu'on regarde \\
\hline $\begin{array}{l}\text { - il y a un «au delà » qu'on } \\
\text { imagine } \\
\text { - le personnage veut trouver } \\
\text { une réponse à ses peurs } \\
\text { - le personnage a envie de } \\
\text { voir de l'autre côté, il désire } \\
\text { - le mur est au bout d'un } \\
\text { jardin, d'une route... }\end{array}$ & $\begin{array}{l}\text { - le mur est plus ou moins solide, plus } \\
\text { ou moins cher, plus ou moins efficace } \\
\text { - le mur est une habitation } \\
\text { - le personnage veut se protéger } \\
\text { - le personnage court un danger }\end{array}$ & $\begin{array}{l}\text { - le mur est souvent } \\
\text { vieux } \\
\text { - quelque chose de } \\
\text { vivant s'oppose au mur } \\
\text { - le personnage accepte } \\
\text { une limite } \\
\text { - le mur est un support } \\
\text { pour voir }\end{array}$ \\
\hline
\end{tabular}




\begin{tabular}{|l|l|l|}
\hline $\begin{array}{l}\text { - on parle de la taille } \\
\text { (longueur / hauteur) }\end{array}$ & $\begin{array}{l}\text { - on parle de sa solidité, de ce en quoi } \\
\text { il est fait } \\
\text { - on parle de ses ouvertures } \\
\text { - on dit ce que le mur laisse } \\
\text { ou ne laisse pas voir }\end{array}$ & $\begin{array}{l}\text { - on dit la qualité du mur } \\
\text { - on parle de sa peinture } \\
\text { - on dit le contraste } \\
\text { entre le mur et le vivant }\end{array}$ \\
\hline
\end{tabular}

\subsection{Une consigne d'écriture floue}

11 Au mois de juin, à la suite de ces travaux qui s'étaient succédé tout le long de l'année scolaire, la maitresse a souhaité voir ce qui en avait pu sédimenter dans l'esprit et la culture des élèves. Dans ce but, elle propose une activité d'écriture à partir d'une consigne très ouverte : «Écris une histoire où il y aura un mur. » Elle espérait obtenir ainsi des productions où elle pourrait saisir quelles configurations du motif auraient été privilégiées, avec quelle netteté ou quelle puissance.

12 Comme cette consigne n'était accompagnée d'aucun travail de remémoration des lectures faites, d'aucune sorte de préparation à l'écriture, les élèves se sont sentis un peu perdus deux d'entre eux n'ont d'ailleurs rien écrit. Les vingt autres ont cependant mobilisé effectivement certains aspects du travail sur le motif du mur, entremêlés d'autres références si bien que le corpus se prête à deux types d'analyse : on peut y lire une sorte de feuilleté de ces multiples sources d'inspiration; on peut aussi lire dans cet ensemble des éléments des configurations naguère explorées. Il est ainsi loisible d'approcher comment fonctionne une interaction entre lectures d'amont et aval de l'écriture quand cette interaction n'est pas pilotée par un projet d'enseignement.

\section{Ce qui subsiste des études faites en amont}

Dans les productions des élèves, le lecteur peut trouver des allusions aux trois configurations - mur qu'on franchit, mur qu'on bâtit, mur qu'on contemple. Le plus souvent, les élèves se sont appuyés sur une seule des trois. Ainsi, 10 élèves (sur 22 élèves de la classe, et 20 productions) ont repris des éléments du motif « mur qu'on franchit », 5 des éléments du motif «mur qu'on contemple» et 2 seulement des éléments du motif " mur qu'on bâtit ». Dans 3 productions, on trouve des éléments se rapportant à plusieurs motifs.

Cependant, il convient de voir plus précisément les éléments qui ont été ainsi sollicités. En effet, outre le script des actions où s'inscrit régulièrement le motif, les élèves se sont employés à mettre aussi en œuvre les valeurs symboliques qu'il peut véhiculer.

\subsection{Le mur qu'on franchit}

15 Le plus souvent, ce sont les aspects techniques qui apparaissent sous la plume des élèves. L. Lionni, dans Tillie et le mur, exposait trois tentatives : le héros essayait d'escalader, de ménager un trou, de longer pour contourner un mur obstacle; l'issue positive était le fruit d'une dernière tentative : le creusement d'un tunnel. Le personnage d'A. Esteban, dans Le Mur, recourt sans succès à l'escalade et au contournement avant de faire pousser 
un arbre sur lequel, après bien des années, enfin grimper. Dans les deux autres ouvrages, le franchissement ne présentait pas de difficultés techniques, mais psychologiques.

Trois productions se contentent d'exposer une série de tentatives vaines qui s'achève sur une qui réussit. Souvent les élèves reprennent escalade, contournement et excavation, mais parfois l'un ou l'autre développe une tentative originale, comme Louis dans son texte ${ }^{3}$ où ses propres aspirations à devenir mécanicien s'entremettent pour imaginer tout un système de poulie et de contrepoids :

Le Mur infranchissable

Il était une fois un jeune homme qui s'appelait Jonathan. Il se promenait tranquillement dans la forêt. Quand il rentra, ses parents faisaient les valises, et disent :

- Nous allons partir en voyage.

- À quelle heure nous partons?

-9 heures du matin.

À la moitié du chemin tout se passait bien, mais un peu plus tard, ils virent un mur qui les bloquait. Ils firent demi-tour et trouvèrent un mécanicien. Ils achetèrent des outils pour casser le mur. Une fois arrivés, ils essayèrent de casser le mur mais le mur était trop solide. Ils réfléchirent : aucune idée. À un moment, Jonathan eut une idée.

- Prendre une échelle !

- On peut aller en acheter.

Donc ils allèrent acheter une échelle. Ce fut dur d'en trouver une assez grande. Ils retournèrent là-bas, le mur n'y était plus. Ils continuèrent et trouvèrent le mur qui s'agrandissait de plus en plus, malheureusement. Vite, ils prirent l'échelle mais ne surent pas comment transporter la voiture. Ils allèrent chercher des objets très lourds.

- Il faut une autre voiture et une chaîne, dit Jonathan.

- Pourquoi faire? répondirent ses parents.

- Vous verrez! Allez me les chercher.

- Jonathan accrocha la chaîne à la voiture, il mit une enclume pour que ça monte

l'autre voiture. Et tranquillement ils allèrent chez eux.

On trouve aussi quelques échos de la lecture du Jardin de Max et Gardénia, de F. Bernard et F. Roca (1998). Aïcha en retient le script : une expédition du héros pour retrouver l'objet de son attachement au-delà du mur ; Lola s'inspire d'une scène de l'album où le crapaud, sorte de vieux sage, sermonne le chat et sa jeunesse intrépide, pour le mettre en garde contre les dangers encourus; Marie ne conserve que l'exercice de la curiosité du personnage pour développer une fantasmagorie personnelle. Cependant, ces allusions sont secondaires, et elles sont insérées dans des univers et des aventures où leur rôle est pour ainsi dire anecdotique. Ainsi le texte d'Aïcha :

Jorge de la Jungle

Un jour les parents de Jorge voulaient faire un voyage et Jorge avait qu'un an. Alors ils sont partis voyager et sur le chemin l'avion s'est écrasé, alors Jorge s'est perdu. Il est tombé dans l'eau, il est tombé sur une bosse et il a voltigé dans l'arbre et des gorilles l'ont baptisé Jorge de la jungle. Alors il a trouvé des amis. Il avait un défaut : c'est quand il prenait une corde, il se prenait le mur. Et il revenait avec des bosses. Et un jour il trouva une fille qui s'appelait Ursula. Il avait un sentiment pour elle, et elle avait son mari qui s'appelait Chef. Qui était derrière le mur. Alors Jorge voulait faire plaisir à Ursula. Alors il dit : « On ira, mais il faudra grimper, courir, sauter, se balancer...

- Bon. J'essaierai.

- Tu es sûre?

- Oui.

- Bon, d'accord. Mais ça va être dur.» 
«- Je croyais que tu ferais un effort, moi.

- Oui, je ferai un effort. "

l'enfant perdu et adopté par des animaux sauvages, la maladresse (sans doute surmontée, vu la répartition des rôles à la fin de la production), la relation amoureuse contrariée, l'encouragement au débutant par l'expert... On voit aussi que l'élève a développé une succession de scènes relativement visuelles : la chute et le rebondissement, les échecs des tarzanades, le dialogue avec Ursula... Le motif du mur, imposé par la consigne, semble seulement fournir l'occasion d'une épreuve qu'il faut surmonter, d'une double épreuve en fait : celle que traverse Jorge au bout de sa corde, et celle qu'affronte Ursula.

\subsection{Le mur qu'on contemple}

19 Cinq élèves ont nourri leur texte de ce motif, ou l'ont pris pour base de leur production. Dans l'album d'Yves Heurté, le personnage était condamné à fixer à longueur de journées le même mur et il développait une méditation qui prenait une forme poétique. Ces poèmes, susurrés, étaient recueillis par les passants, étaient publiés, devenaient une œuvre célèbre. Pour rendre compte de ce dépassement de l'humaine condition, les élèves ont imaginé un mur «magique», doté de pouvoirs extraordinaires. De manière plus inattendue, deux élèves ont coloré leur production d'une dimension religieuse. Ainsi le texte de Julien :

Le mur qu'on regarde

Il était une fois un jeune garçon qui vivait dans une ferme. Il avait un mur dans sa chambre et tous les soirs il faisait ses prières, devant son mur. Un jour il était obligé de se marier. Deux semaines plus tard quand il est marié, il allait voir son mur tous les jours avec sa femme. Sa mère lui demandait toujours pourquoi il aimait ce mur. Mais il ne disait jamais. Un jour il était si content, il a dit : «Maman, je vais te dire pourquoi j'aime ce mur : c'est parce qu'il m'a porté chance pour ma vie. »

Dans cette production, il n'est bien sûr pas impossible d'apparenter l'attachement au mur familier à une fixation à l'enfance et ces actions de grâce réitérées à une sorte de «méthode Coué » pour endurer un état matrimonial (il était obligé de se marier). Cette interprétation ravageuse ne rend sûrement pas compte de la complexité d'un motif qui marque le dépassement : de l'état d'enfance à l'état d'adulte, du silence à l'expression, de l'humanité vers la transcendance.

\subsection{Le mur qu'on bâtit}

21

Fort peu d'élèves ont sélectionné cet aspect du motif, deux seulement en fait. À la question de ce qui motivait cette désaffection, la classe a répondu par la lassitude d'un conte trop bien connu. Ceux qui le mettent en œuvre ont essentiellement retenu l'ambivalence du mur, lequel peut protéger aussi bien qu'enfermer dans une sorte de prison dorée. Dans le texte de Céline, la tension se réduit grâce à un accroissement heureux de son territoire :

Il était une fois une sorcière qui s'appelait Anna. Elle n'aimait pas les gens. Mais elle avait des voisins qui venaient souvent la voir. Elle était très en colère, des fois elle s'enfermait chez elle. Mais elle s'ennuyait et elle ne pouvait pas supporter d'être enfermée. Un jour elle eut l'idée de construire un mur autour de son jardin. Depuis elle vit heureuse. 


\subsection{Minceur de l'influence des textes lus}

22 Au total, ce que les élèves retiennent du motif, ne relève en rien de ces invariants qui avaient été formalisés : on ne trouve pas d'au-delà imaginé derrière les murs à franchir, ni paresse ni assiduité dans la réalisation de murs à construire, aucune vie microscopique à observer dans les murs qu'on contemple. Comme on pouvait s'y attendre, est repris essentiellement le script d'actions dans lequel il peut s'inscrire. Cependant, on ne peut nier que les élèves recueillent quelque chose des valeurs symboliques qu'ils avaient perçues dans les moments de lecture. On trouve ainsi une fascination pour l'ingéniosité technique (qui n'était pas apparue explicitement dans la phase de lecture), le traitement d'un mur qui dissimule un danger à craindre, un objet de désir ou une surprise plus ou moins heureuse, ou encore la confrontation initiatique avec un mur qui constitue une épreuve à subir pour grandir, s'affirmer, s'accomplir. On trouve aussi l'ambivalence des protections où l'on risque de s'enfermer, et la dimension transcendante qui anime toute contemplation.

Il ne faudrait cependant pas forcer le trait, ces éléments - on l'a vu - sont peu marqués, peu développés. Il y a, certes, de la lecture à l'écriture une déperdition de la finesse interprétative... On peut le comprendre comme la confirmation de ces deux points bien connus : d'une part, l'appui sur le collectif, entre émulation et soutien, avait permis d'élaborer une perception fine et aigüe des ensembles proposés; d'autre part, le travail d'écriture, solitaire, monologique, d'une complexité pluridimensionnelle et d'une objectivité irrécusable, ne manifeste pas chez le même élève des compétences aussi assurées que celles qu'il donne à voir quand il coopère dans des tâches tâtonnantes d'interprétation. Programmer à destination d'un lecteur un travail interprétatif suppose une maitrise des effets à produire et, «de la lecture à l'écriture, le transfert n'est pas direct » (Tauveron \& Sève, 2005). Ici, ce qui en ressurgit ne semble pas de l'ordre du calcul mais plutôt d'un effet de rémanence qui dépend étroitement des complexions psychoaffectives singulières de chacun des élèves. Sans doute est-ce pour cette raison que deux élèves ont renoncé à entrer dans une tâche de production dont ils sentaient les enjeux, et aussi combien elle excédait leurs compétences.

\section{Le feuilleté des textes}

Quand il y a hésitation entre deux configurations, la référence à un mur fonctionne comme une obligation qui opère une contrainte sur un imaginaire plus personnel. Ainsi le texte de Quentin :

Il était une fois un oiseau qui s'appelait Gugus. Il construisit un mur pour que Baba le chat l'attrape pas. Mais il y avait la buse Binocle.

Il était fermé par un mur qu'il voulait franchir. Mais il ne pouvait pas parce que Baba et Binocle le guettaient. Un jour Baba dit: «Je l'aurai, cet oiseau!» Alors Gugus voulut s'en aller, mais Binocle le poursuivit. Donc Gugus retourna dans son nid.

Le script fondamental de cette histoire, la double poursuite par un chat et une buse, semble imité de Mangetout et Maigrelet de C. Boujon (1992). En effet, dans ce récit, deux oiseaux sont chassés l'un par un chat et l'autre par un aigle. Quentin, interrogé sur ce point, a confirmé cette source d'inspiration: cet album avait été travaillé l'année 
précédente en classe de CE1, et comme Quentin l'avait fort apprécié, ses parents le lui avaient offert.

La mention d'un mur à construire, puis d'un mur à franchir, engendre de la perplexité. S'agit-il du même mur qui enferme autant qu'il protège, qui crée un enfermement après qu'il a servi de protection? L'emploi dans les deux cas d'un déterminant indéfini fait obstacle à cette interprétation, mais l'on sait qu'à ce niveau d'enseignement la maitrise de l'opposition défini/indéfini est souvent approximative... S'agit-il de deux murs distincts, l'auteur a-t-il voulu mobiliser successivement les deux motifs? Mais lors de la seconde mention d'un mur, l'obstacle n'est pas tant dans la présence du mur que dans la présence des ennemis bien identifiés et que le mur ne dissimule pas... Notre hypothèse est plutôt que l'élève avait en tête une situation de conflit entre proie et prédateurs, avec ses développements potentiels en forme de quête d'un refuge et de course poursuite, et qu'il a seulement voulu se «mettre en règle» avec la consigne donnée. D'ailleurs, le vocabulaire employé (le verbe franchir n'appartient guère au vocabulaire de la langue enfantine) signale une insertion volontaire.

La lecture de cette production à elle seule nous incite donc à identifier dans l'ensemble du corpus les traits qui signalent des éléments issus de souvenirs de lecture, éléments de formats divers depuis un script d'actions qui court sur l'ensemble du texte jusqu'au mot isolé, éléments qui s'offrent à des procédures diverses, depuis un emprunt discret jusqu'à, parfois, la simple copie.

\subsection{Variation}

Certains élèves restent d'une grande fidélité à l'un des textes. Ainsi le texte de Benjamin :
La Petite Souris Henri
Il était une fois des souris qui habitaient dans un champ. Elles étaient sept. Il y en avait une qui s'appelait Henri. Un jour, comme il y avait un mur dans le champ, Henri décida de voir ce qu'il y a derrière le mur. Elle essaya par tous les chemins. Il essaya avec une échelle : elle était trop courte. Il essaya de l'escalader : il n'arrivait pas à s'accrocher. Un jour elle a même essayé de dire : «Est-ce qu'il y a quelqu'un derrière le mur? » Personne ne répondit. Alors un jour elle eut l'idée de creuser un tunnel.

Sans reprendre exactement les expressions du texte, il suit pas à pas l'œuvre de Leo Lionni. Il reformule avec ses propres mots les étapes du récit (détermination du héros, prise de décision, épreuves, invention d'une solution) et les différentes tentatives de la jeune souris. Sa production parait ainsi un résumé. Pourtant, il modifie le nom du héros, il pousse l'anthropomorphisation de l'animal en le dotant d'une échelle. On a donc l'impression que l'élève ne s'est pas hissé à la hauteur proposée par le réseau des textes lus, ou qu'il y a renoncé, et qu'il a préféré s'appuyer sur le texte qu'il s'était le mieux approprié. Il a seulement mis de lui-même par ses ajouts ou ses modifications et a ainsi produit une variation.

30 Nous définissons donc la variation comme l'inscription du scripteur dans le sillage d'un texte qu'il élit comme matrice de son propre texte. La variation peut osciller entre copie et reformulation, mais elle se coule dans un projet qui n'est pas authentiquement celui du scripteur. On se souvient que dans l'Athènes antique le recours à des avocats était proscrit et que les citoyens devaient présenter eux-mêmes leur plaidoirie. Comme ils n'avaient pas tous des talents oratoires bien assurés, ils faisaient parfois appel à des écrivains patentés pour leur fournir un discours qu'ils apprendraient par cœur, qu'on 
appelle des logographes. Ceux-ci, pour fournir à leur client un texte qu'il puisse prétendre le sien avec quelque vraisemblance, devaient se conformer à un caractère et à un mode de pensée qui n'étaient pas de leur éthos propre. Ici, comme Lysias adoptait la personnalité et jusqu'aux tics de langage de son barbier engagé dans un méchant procès, Benjamin endosse une posture qui n'est pas exactement la sienne, mais celle qu'il suppose attendue par la maitresse et légitimée par l'aval éditorial.

\subsection{Emprunt}

Dans beaucoup de productions, les élèves mobilisent un univers de référence qui fait partie de leur culture personnelle. Nous avons dit que Quentin reprend des éléments de Mangetout et Maigrelet ; plusieurs de ses camarades, comme Aïcha, Lola, Jibril ou Gaëtan, sollicitent un film d'animation pour enfants qui était d'actualité à l'époque de ce travail, Le Château des Singes de Jean-François Laguionie. Maëva insère le mur prescrit dans un univers de conte merveilleux, Augustin dans une espèce d'enquête menée par un Hercule Poirot très singulier ${ }^{4}$. Outre le script, ce sont de mêmes personnages, éléments de décor, ambiance qui constituent la matière de leurs récits.

Dans notre corpus, le texte de Justine parait remarquablement original, voire spectaculaire, parce que ce fond d'emprunt ne relève ni de films ou de séries télévisuelles en vogue ni d'une figuration de la vie quotidienne au réalisme hésitant, mais qu'il semble provenir d'un substrat mythique plus mystérieux. Voici ce texte :

Le Mur magique

Un jour une jument qui s'appelle Petite Perle mais un jour qu'elle était en balade,

elle demanda au cavalier de rentrer. Il était d'accord. Quand elle rentra à l'écurie, elle était enfermée entre quatre murs. L'un était différent des autres. Un soir, un ange sortit du mur. L'ange s'appelait Magellan et il lui donna un poulain.

Ici, il se déploie sans doute une rêverie sur ce que les psychologues appellent la scène originelle, nourrie d'emprunts aux scènes d'Annonciation ou aux mythes de naissances virginales, et transposée dans un univers équestre. Le nom de Magellan, qui se détache de ce fond, excite alors un travail d'interprétation : que vient faire ici le nom du navigateur, ou du périlleux détroit? Est-ce un pur jeu d'assonance (lãz / mazelã) qui insiste sur son existence séraphique? Sans doute le texte ne permet pas d'avancer de solides hypothèses, il est trop succinct, et il s'agit probablement d'un hasard heureux, de ce qu'on appelle communément une trouvaille. Mais le contraste entre un fond d'emprunt que partage le lecteur et une notation inattendue met en branle une activité proprement lectorale. Sans doute il serait aventureux d'appliquer à l'écriture enfantine les analyses qu'un Iser (1985) élabore pour rendre compte de l'« effet esthétique» des textes experts, cependant on peut voir ici à l'œuvre un "répertoire» qui vient actualiser et interroger une configuration (mé)connue.

Nous définissons donc l'emprunt comme l'utilisation d'une matière fictionnelle telle que l'enfant a pu se l'approprier, dont il a fait la matière même de son imaginaire. Même si cette matière combine script d'actions, éléments de décors, caractérisation des personnages, traits de langage, même si l'on a affaire à un compost d'éléments d'origines diverses et hétérogènes, elle apparait comme un ensemble unifié dans les mouvements complémentaires de reconnaissance d'une part et de perception d'un inattendu d'autre part qui animent à eux deux toute lecture. 


\subsection{Remploi}

$35 \mathrm{Au}$ rebours de ces emprunts susceptibles de passer inaperçus tant ils sont naturalisés dans l'univers fictionnel par la puissance intégrative du récit produit, les productions enfantines donnent parfois à lire certains fragments qui s'insèrent mal dans le tissu déjà constitué. Ainsi le texte de Théo :

Le mur sans fin

Un jour un chat passa devant le mur. Il eut envie de voir ce qu'il [y avait] derrière mais il pensait que ce mur était sans fin. Il prit une décision et dit : «Je vais essayer de le grimper ». Il a essayé pendant des heures sans y arriver. Un jour il eut l'idée de prendre une échelle, une grande échelle. Mais comme c'était un chat, il ne pouvait pas la prendre. Il alla voir son maître et lui dit : « Mon maître, est-ce que tu peux me mettre l'échelle sur ce mur?» Le garçon dit: «Oui ». Donc le jeune homme mit l'échelle contre le mur et emporta sur le mur le chat, alors le chat vit ce qu'il y a derrière, mais nous, nous savons pas ce que le chat a vu.

Théo, comme Benjamin, reprend pour l'essentiel la matière du récit de Leo Lionni. Il recrute comme héros un chat au lieu d'une souris, et il esquive un anthropomorphisme poussé en mettant en scène le maitre de l'animal. Ce qui est singulier, c'est l'insertion finale. Ici, Théo reprend la sorte de prétérition qui clôt le récit d'A. Esteban : « $\mathrm{Il}^{5}$ atteignit enfin la cime, Il embrassa du regard le nouveau paysage. On ignore ce qu'Il découvrit de l'autre côté ; une autre mer peut-être ou bien de verts bocages, peut-être seulement une terre stérile. » Le recyclage est manifeste, mais comme il n'est pas fait une claire mention d'espérances longuement nourries et qu'il n'est indiqué qu'une "envie de voir», la frustration du lecteur n'est guère ludique, ni justifiée comme elle pouvait l'être par le texte d'A. Esteban. Ici, l'enfant a trouvé que c'était une "bonne idée» et a tenté de l'importer dans son propre texte.

Pour désigner ces morceaux insérés non sans faire quelque accroc à la dynamique d'ensemble, nous avons recours au terme de remploi, tel qu'il est utilisé en architecture. Dans ce domaine, il désigne l'utilisation d'un élément déjà existant, avant même qu'ait émergé le projet du bâtiment à construire. Il s'agit souvent d'un fragment de monument prestigieux récupéré parmi des ruines. Plusieurs églises de Rome contiennent ainsi des morceaux - colonnes, chapiteaux, frises ornementales... - qui proviennent d'antiques temples païens. Il ne manque pas dans les villes anciennes de maisons du XVIII ${ }^{e}$ siècle où l'on identifie un cadre de fenêtre gothique, voire une arcature romane. À chaque fois, c'est le décalage dans le style ou dans les époques qui signale l'élément réutilisé. De même, dans l'écriture enfantine, un remploi peut se laisser détecter par un décalage stylistique, par une incongruité ou par une sorte de diglossie quand l'élève manie une langue par trop inhabituelle sous la plume d'enfants de son âge.

\subsection{Le dynamisme de l'écriture enfantine}

$\mathrm{Au}$ regard des emprunts que le lecteur ne détecte qu'à la condition de connaitre la matière empruntée, l'obligation d'insérer la mention d'un mur, loin de fonctionner comme une consigne oulipienne et créative, a pu paraitre arbitraire, profondément étrangère à la dynamique d'engendrement du texte. En quelque sorte, dans à peu près la moitié des productions recueillies, le surgissement d'un mur donne plutôt l'impression d'un remploi, corps étranger au dynamisme. Ainsi, le texte de Léna : 
Le petit garçon qui cherchait un abri

Il était une fois un petit garçon qui s'appelait Tomy et il habitait dans une cabane au fond de la forêt. Un jour il faisait un temps superbe. La mère de Tomy lui dit : « Estce que tu peux aller me chercher du pain tout en bas de la forêt?» Tomy lui répondit : «D'accord. » Il prit son panier et descendit tout en bas de la forêt. Dès qu'il trouva la route, il se mit à pleuvoir, et plus il allait vers la boulangerie, plus il pleuvait. Même il tombait de la grêle. Tomy se dit : « Il faut que je trouve un abri. » À un moment il trouva une sorte de préau. Il se précipita sous le préau et attendit une heure. Après il se précipita dans la boulangerie et demanda du pain, un sandwich, une bouteille de coca-cola et des bonbons. La dame lui dit: «Ça fera quinze euros. » Tomy lui dit : «Je n'ai que douze euros. » La dame dit : «J'attends. Va dehors, débrouille-toi. » Juste à ce moment-là un monsieur passait dans la boulangerie et dit: "Qu'est-ce qu'il y a, bonhomme?" Tomy répondit: «Je dois quinze euros à cette dame et je n'ai que douze euros. » Le monsieur lui dit : «Tiens, je te donne trois euros.» Tomy lui dit: «Merci, Monsieur.» Le monsieur prit le panier où il $\mathrm{y}$ avait du pain, un sandwich, une bouteille de coca-cola et des bonbons. Après, quand ils sortirent de la boulangerie, il faisait le même temps que ce matin. Tomy était très content. Dès qu'ils sont arrivés chez lui, sa maman lui dit : "Qui c'est, ce monsieur? » Tomy lui raconta tout ce qui s'était passé. À la fin, quand il dut partir, il dit: "Je vais vous donner mon numéro de téléphone, mon adresse, mon nom et mon prénom. » Il donna tout ça et tous les jours Tom venait voir ce qui se passait. Tom était le monsieur. Un jour, Tom raconta que dans son jardin, il y avait un mur qui avait poussé et même il parlait. Tomy dit : « Je te crois pas. » Il dit : «Viens voir. » Et il lui montra le mur et il le vit parler. Et comme il n'avait pas de copain, ça lui en faisait un. Et un jour, le mur lui dit : «Comme je suis magicien, je vais te faire plein de copains, et tout... »

39 Cette production, on le voit, n'émane pas d'une élève de 9 ans qui serait fragile. Au contraire, la longueur remarquable, la lisibilité assurée, les petites notations réalistes qui enrôlent le lecteur font la preuve de compétences déjà bien installées. Cependant, le dernier épisode, celui qui répond réellement à la consigne, apparait comme un remploi, superflu, comme étranger au reste du texte.

Certaines maladresses, courantes dans l'écriture enfantine, permettent de former quelques hypothèses sur les dynamismes qui ont présidé à son engendrement. Ici, le souci rétrospectif d'apporter une information importante ( Tom était le monsieur») signale que la prise en compte du lecteur à venir n'est pas au cœur de l'écriture. En revanche, le jeu de reprise sur le prénom des personnages (comparez Tom à Tomy) vient confirmer un enjeu perceptible dès la mention de la cabane et de la forêt (et déjà le titre) : il s'agit du script de «l'enfant perdu » et de la quête d'une puissance - paternelle? - capable de rassurer, de garantir l'alimentation et - en l'occurrence - de se soucier aussi de la mère. L'ensemble manifeste une grande capacité d'intégration des divers développements.

41 Par ailleurs, la présence de dialogues imités de la vie quotidienne est la marque d'un appui non pas seulement sur des modèles textuels mais sur une production langagière fictionnalisante, comme la décrit F. François (2004). Pour être plus précis, sans entrer dans l'épineux débat sur la validité d'une instance «narrateur» dans le récit et ses éventuelles marques linguistiques ${ }^{6}$, l'élaboration d'un dialogue qui imite une quotidienneté - si fréquente dans les productions enfantines - construit et, sans doute, entretient un creuset où le « je » du scripteur s'accommode aux « je » de ses personnages, se distinguant ainsi du «je » de l'enfant. Dans ce creuset, modèles textuels, répertoire lexical, connaissances encyclopédiques et enjeux psychoaffectifs peuvent se nouer, se mêler, s'unifier en un dialogisme qui garde sa part d'énigme. Un tel pas de côté qui suspend l'urgence des interlocutions orales, ou plutôt qui la transpose et la transforme, 
ouvre l'espace d'un langage intérieur et constitue le lieu d'où le récit risque de donner forme à une pensée.

La préoccupation de l'injonction sociale conduit l'élève à répondre in fine à la consigne : Léna ajoute à son histoire l'épisode merveilleux d'un mur qui pousse, qui parle, qui devient un "copain " et qui possède des pouvoirs. Elle remploie ce faisant la matière essentielle de l'album dans lequel le mur permettait au personnage, condamné comme " au piquet ", d'échapper à la solitude parce qu'il offrait à sa contemplation une lézarde peuplée d'insectes. Mais, et cela ne contribue pas peu à l'impression de grande maitrise, cette matière, elle se l'approprie, la transforme. Ici encore, la présence de dialogues peut être symptomatique d'une sorte de théâtre intérieur, d'une instance qui permet la refonte, même si reste visible une suture entre cette adjonction et le morceau qui la précède.

Si l'on reconnait une pertinence à ce commentaire, l'on mesurera ce qui sépare une production comme celle de Léna de celle que nous avons présentée comme "variation ». On peut faire l'hypothèse que Benjamin, qui reprend le développement de Tillie et le mur, n'a guère recours à ce " pas de côté » et ne cherche pas ce qu'il pourrait avoir «à dire » en réponse à la consigne. En quelque sorte, comme ce ne semble pas si rare dans les classes, le projet actualisé dans l'écriture du texte n'est pas celui du scripteur, ce n'est pas celui de l'enfant Benjamin; c'est celui de la maitresse tel que Benjamin se le figure. Benjamin adopte une posture de logographe, il se met en règle avec la prescription scolaire, il ne s'engage pas dans l'aventure de l'écriture.

Par contraste, on mesurera aussi comment Léna opère une sorte de lissage quand elle intègre finalement un élément qui réponde à la consigne. De fait, elle sélectionne parmi les textes du corpus et utilise l'épisode qui entre le mieux en résonance avec le script de l'« enfant perdu " qu'elle avait choisi de développer. Ainsi, c'est un lecteur dans la connivence de la consigne et des œuvres lues en amont qui détecte ce qui peut paraitre un emprunt, un lecteur non affranchi le prendrait pour l'amorce d'un second développement.

\section{Bilan et perspectives}

45 À partir de ces textes d'enfants, parce qu'ils ont été produits sans aides particulières et sans que soit clairement identifiable un attendu de la maitresse, il semble possible de retenir quelques caractéristiques de l'écriture enfantine.

Comme dans les premières tentatives d'écriture autonome chez les élèves de quatre ou cinq ans, le recours à la copie peut constituer en soi un projet cohérent. Se tenir dans la position où s'est tenu un énonciateur légitime peut suffire pour engendrer un texte. Les auteurs de variations sont souvent - en tout cas dans cette classe - des élèves que la maitresse juge fragiles ou timides ; il semble ainsi qu'ils évitent une prise de risque, qu'ils évitent d'avoir à construire une position d'énonciation et à élaborer la distinction entre un «moi qui vis» et un "moi qui écris", qu'ils mettent le premier à l'abri des incertitudes du second. Sur un autre plan, de même que les très jeunes enfants s'émerveillent de tenir au bout de leur crayon le pouvoir d'évoquer un chauffe-eau ou une moto quand ils en ont recopiés le nom ${ }^{7}$, de même l'on constate chez les élèves un plaisir réel à orner leur texte de trouvailles glanées ici ou là, quitte à ce que ces remplois ne passent pas totalement inaperçus. 
47 Mais l'essentiel - et nous rejoignons ici les positions que défend D. Bucheton (2014) parait bien se jouer dans l'émergence progressive d'une intériorité « réflexive » où l'élève trouve ce qu'il peut avoir à dire et donc élabore un projet qui soit réellement sien dans le tissage et la confrontation avec tout ce qu'il a lu, vu, entendu, avec tout ce qu'il a pensé grâce à cette matière verbale, avec tous les modèles, stéréotypes et règles que, les fréquentant à suffisance, il a incorporés à ses propres ressources langagières.

S'il est vrai que les premiers balbutiements d'écriture se jouent ainsi entre copie, variation, emprunt et remploi, et avant même de nourrir l'ambition d'amener les élèves à une pratique experte telle que les modèles de John-R. Hayes et Linda Flower, ou d'autres, la décrivent et telle que la visent les dispositifs didactiques inspirés du groupe EVA, il convient sans doute d'imaginer les moyens d'accompagner d'abord et de soutenir les prises de risque que constitue l'élaboration de cette intériorité réflexive. C'est ainsi qu'en nous appuyant sur la pratique de la variation, attestée dans ce corpus mais bien perceptible dans d'autres, nous avons imaginé un dispositif d'enseignement: nous proposons avec une certaine régularité à des classes deux versions d'un même conte, avec la consigne: "Écris l'histoire à ta manière." Les productions recueillies sont alors regardées par la classe sous l'angle du risque pris, depuis un simple centon de citations recopiées jusqu'à, parfois, une réélaboration complète des enjeux et la réfection de la fiction entière au service d'un projet authentiquement personnel. D'eux-mêmes, en dehors de tout jugement de valeur énoncé explicitement mais souvent en s'appuyant sur le miroir que leur tendent les camarades, les élèves perçoivent vite qu'il est plus intéressant de risquer le " pas de côté »: les travaux d'écriture se trouvent alors pris dans la construction d'une forme de narcissisme de scripteur, les jeunes auteurs acquièrent vite le sentiment qu'ils savent écrire et inaugurent une pratique de recyclage de leurs trouvailles antérieures, prémisse de la construction d'un style.

\section{Conclusion}

Les adultes ne lisent pas les textes enfantins en adoptant l'attitude de "supporters » qu'ils adoptent spontanément pour encourager les tâtonnements de l'apprentissage de la parole. Les maitres eux-mêmes, bien souvent, demandent d'écrire " des textes » mais se saisissent d'emblée d'un crayon pour annoter, si bien qu'ils lisent de facto une succession de phrases. Il semble qu'ils conduisent souvent les dispositifs d'enseignement de l'écriture en réglant leurs interventions sur un "texte fantôme », celui que décrivaient les « aides à l'écriture » qu'ils ont dispensées, celui qu'ils souhaiteraient voir écrit, celui qu'ils écriraient peut-être ${ }^{8}$ eux-mêmes en réponse à la consigne. Ce faisant, ils présupposent les compétences à écrire qu'il s'agit justement de faire acquérir. Or ces compétences, si on veut les définir non comme maitrise linguistique mais comme compétences langagières, paraissent prendre consistance comme font les compétences des jeunes enfants à parler : copier les propos tenus par des locuteurs adultes, remployer des formulations efficaces, emprunter à l'envi et peu à peu stabiliser, sous l'œil bienveillant des adultes, une position d'où énoncer son identité, ses aspirations, ses émotions... Aussi, avant que de demander aux élèves de contrôler et régler leurs productions, il importe de leur présenter l'écriture comme un espace de choix, de détermination et de réflexion, somme toute, comme un espace où déployer une intelligence épilinguistique. 


\section{BIBLIOGRAPHY}

BERNARD, F. \& ROCA, F. (1998). Le jardin de Max et Gardénia. Paris : A. Michel jeunesse.

BERNOS, C. (1986). Volubilis et les trois jardins. Moulins : Ipomée.

BORÉ, C. (2010). Modalités de la fiction dans l'écriture scolaire. Paris : L'Harmattan.

Boujon, C. (1992). Mangetout et Maigrelet. Paris : L'École des loisirs.

BUCHETON, D. (2014). Refonder l'enseignement de l'écriture. Paris : Retz.

ESTEBAN, A. (1991). Le Mur. Paris : Syros.

FRANÇOIS, F. (2004). Enfants et récits. Mises en mots et « reste». Villeneuve d'Ascq : Presses

universitaires du Septentrion.

HEURTÉ, Y. (1998). Le Livre de la Lézarde. Paris : Seuil Jeunesse.

ISER, W. (1985). L'acte de lecture. Théorie de l'effet esthétique. Sprimont : P. Mardaga.

KiShida E. \& futAmata E., (1993). Les Trois Petits Cochons. Paris : L'École des loisirs.

LIONNI, L. (1989). Tillie et le mur. Paris : L'École des loisirs.

MAGGI, S. (1998). Les Trois Petits Cochons. Ingersheim : SAEP.

MEN. (1992) La maitrise de la langue à l'école. Paris : CNDP.

PINGET, R. (1984). Le Harnais. Paris : Minuit.

SÈvE, P. (2004). « L'aide à la lecture ». In : Tauveron, C. La Lecture et la culture littéraires au cycle des approfondissements. Versailles : Scéren, p. 91-97.

- (2005). «Évaluer les écrits littéraires des élèves », Repères 31, p. 29-52.

- (2014). « Profusion et confusion dans l'écriture enfantine ». Forumlecture. En ligne : http://

www.leseforum.ch/myUploadData/files/2014_3_Seve.pdf.

STEHR, F. (1997). Les Trois Petites Cochonnes. Paris : L'école des loisirs.

TAUVERON, C. \& SÈVE, P. (2005). Vers une écriture littéraire, Hatier.

\section{NOTES}

1. Il n'est pas jusqu'aux textes officiels qui ne témoignent parfois d'une forme de condescendance: «Encore faut-il accepter avec bienveillance des écrits peu originaux, démarquant parfois fortement des textes présents dans la classe et veiller à les considérer comme de "véritables" productions » MEN (1992: 158).

2. Classe de Véronique Sudre, PEMF à l'école Nestor Perret de Clermont-Ferrand.

3. Dans la mesure où l'orthographe n'est pas prise en compte dans nos commentaires, nous avons cru pouvoir dispenser le lecteur de l'effort supplémentaire qu'imposeraient des choix orthographiques souvent incongrus. En revanche, nous n'avons rien modifié des éventuels alinéas. 
4. Il a été rendu compte de ce texte dans la revue en ligne Forumlecture sous le titre «Profusion et confusion dans l'écriture enfantine » (Sève, 2014).

5. Dans cette œuvre, le personnage n'a pas de nom, il est seulement désigné par le pronom affecté d'une majuscule.

6. Sur ce point, voir le résumé qu'en fait C. Boré (2010: 39-62).

7. Mais aussi certains adultes : "Redire scabieuse acacia melilot, et voilà l'été sur ma page.» (Pinget, $1984: 46)$.

8. Contrairement à ce qui se passe quand ils donnent un exercice de mathématiques, il parait rarissime que les maitres s'obligent à répondre eux-mêmes aux consignes d'écriture qu'ils donnent.

\section{ABSTRACTS}

A body of texts written by some around 9 years old children has been studied. These texts were produced after reading texts in which the wall motif is dominant. However the instructions were made so the pupils couldn't identify what was wanted from them, and thus each and every one of them had to elaborate an original writing project to answer them. In these productions what we see first is the remains of the former readings, and we consequently analyze the different forms the reference to read texts take in singular writing projects. From this analysis we will open up a perspective towards an original teaching dispositive.

Il est mené l'étude, illustrée d'exemples caractéristiques, d'un corpus de textes d'enfants d'environ 9 ans. Ces textes ont été produits à la suite de la lecture d'un réseau d'œuvres centré sur le motif du mur. Cependant la consigne est telle que les élèves n'étaient pas en situation d'identifier clairement quelle espèce de récit était attendue, si bien qu'ils ont dû élaborer chacun un projet d'écriture original pour y répondre. Dans ces productions, on repère d'abord ce qui reste des lectures faites en amont de l'écriture, et l'on analyse les diverses formes que prend la référence aux textes lus dans les projets d'écriture singuliers. Sur la base de cette analyse, on ouvre une perspective vers un dispositif original d'enseignement.

\section{INDEX}

Mots-clés: écriture, enseignement, texte, école primaire

Keywords: writing, teaching, text, primary school

\section{AUTHOR}

\section{PIERRE SÈVE}

Université Blaise-Pascal, ACTé, EA 4281, ESPÉ Clermont-Auvergne, F-63000, France 